\title{
Economic production of VAc and VEOVA 10 emulsion polymer using factorial design
}

\author{
I.A. Sabbah \\ Faculty of Science, \\ Organic Chemistry Department, \\ Cairo 11651, Egypt
}

\author{
Tamer A. Mohamed* \\ Mechanical Engineering Department, \\ The British University in Egypt, \\ Cairo 11837, Egypt \\ Email: tamer.mohamed@bue.edu.eg \\ ${ }^{*}$ Corresponding author
}

\section{H.E. Nasr}

Polymer and Pigment Department, National Research Centre, Dokki, Cairo 12311, Egypt

\section{Sh.E. Emam}

German-Lebanese for Industries, Cairo, Egypt

Email: Sherifemam3@hotmail.com

\begin{abstract}
Emulsion polymerisation of vinyl acetate (VAc) and vinyl ester of versatic acid (VEOVA 10) was carried out using different feed composition ratios. The copolymer emulsion was characterised by measuring the morphological properties (average particle diameter, $D$ ) by using the transmission electron microscope (TEM); minimum film forming temperature (MFFT) and filtration residue (ppm). The results of the study indicate that the average particle diameter of emulsion $(D)$ decreases with the increase of the amount of VEOVA 10 content (and subsequently decreases the amount of VAc), also the ppm and the MFFT is decreased. These results were illustrated using response surface methodology and contour plots. In this paper, the economic production of VAc and VEOVA 10 emulsion polymer was studied to ensure the best performance of all properties at minimum cost utilising multiobjective optimisation methodology.
\end{abstract}

Keywords: emulsion polymerisation; vinyl acetate; VAc; vinyl ester of versatic acid; VEOVA 10; factorial design; response surface methodology; design expert software. 
Reference to this paper should be made as follows: Sabbah, I.A., Mohamed, T.A., Nasr, H.E. and Emam, Sh.E. (2015) 'Economic production of VAc and VEOVA 10 emulsion polymer using factorial design', Int. J. Collaborative Enterprise, Vol. 5, Nos. 1/2, pp.89-101.

Biographical notes: I.A. Sabbah is a Professor of Organic Chemistry at the Department of Organic Chemistry, Faculty of Science, Al-Azhar University, Cairo, Egypt. He had many publications in this area and supervised many MSc and $\mathrm{PhD}$ students in the same area of expertise.

Tamer A. Mohamed is an Associate Professor of Industrial Engineering, Mechanical Engineering Department, Faculty of Engineering, The British University in Egypt, Cairo, Egypt. His area of research is production optimisation. Most of his research is applied research.

H.E. Nasr is a Professor of Polymer Chemistry at the Polymer and Pigment Department, National Research Centre, Dokki, Cairo, Egypt. She had many publications in this area and supervised many MSc and $\mathrm{PhD}$ students in the same area of expertise.

Sh.E. Emam is the R\&D Manager at the German-Lebanese for Industries Company. He has about 20 years of experience in the area of emulsion polymerisation and coating.

\section{Introduction}

Emulsion polymerisation is one of the most important techniques for the production of aqueous dispersed polymers, also called Latexes, from an industrial point of view (Lovell and El-Asser, 1997; Urban and Takamura, 2002). A variety of polymerisation methods have been used including emulsion polymerisation (Zhao et al., 2006) and dispersion polymerisation (Kondo et al., 1994; Niu et al., 2010). Polymer dispersions are used to protect metal, wood and any substrate against water and microorganisms and are used as a binder for pigment and filler to make final paint (Urban and Takamura, 2002; Fitch, 1997).

Emulsion polymerisation is a process of great industrial importance. It finds applications in the manufacture of a wide range of products such as paints, adhesives and production of coatings and other synthetic materials (Allen and Bevington, 1989). Emulsion copolymerisation of vinyl acetate (VAc) with vinyl ester of versatic acid (VEOVA 10) has the potential to outperform VAc - acrylic polymers due to random copolymerisation nature of the first set. Branched vinyl esters (VEOVA 10) are resistant to hydrolysis, both in monomers and polymers state due to their neighbouring group steric effect. VAc co-polymers with VEOVA 10 monomers have a hydrolytic stability with increasing concentration of VEOVA 10. The hydrocarbon chains from VEOVA 10 monomer shield neighbouring VAc groups from hydrolytic attack by the use of water. One monomer unit of VEOVA 10 protects two to the three VAc units. This industry has grown into a multi-million dollar industry today. The global market in the year 2000 for the synthetic latex polymers was estimated to be about 14.10 billion dollar (URL: http://www.klinegroup.com/brochures/v359/brochure.pdf). This paper statistically studies the controllable factors that affect the emulsion co-polymerisation of VAc-VEOVA 10 
polymer at different feed composition ratios. The obtained co-polymer will be characterised with respect to morphological properties (average particle diameter, $D$ ) by using the transmission electron microscope (TEM); minimum film forming temperature (MFFT) and filtration residue (ppm). Also the economic production of VAc-VEOVA 10 emulsion polymer which gives the best performance of all properties at minimum cost is studied.

The following sections explain the experimental preparation of the specimens, followed by factorial design. Section 4 discusses the statistical analysis of the results including response surface methodology. The optimisation of the VAc-VEOVA production is explained in Section 5 followed by the conclusion.

\section{Experimental preparation and methodology}

\subsection{Materials}

Reagent grade VAc was supplied by Aldrich Co. VEOVA 10 with more than $99 \%$ purity was supplied by Shell Chemicals Co. The inhibitors in both monomers were removed by using inhibitor remover (disposable column is used for removing hydroquinone). The treated monomers were stored at $-2{ }^{\circ} \mathrm{C}$ until used. Potassium per sulphate (KPS, 99\%, supplied by Aldrich), sodium lauryl sulphate (SLS 98\%, supplied by Aldrich), sodium meta-bisulphite (95\%, supplied by Aldrich) and 2.2-azobis 2-methyl propionitrile ( $98 \%$, supplied by Aldrich) were used in the copolymerisation process of our experiment. The water used was deionised throughout the experiment.

\subsection{Polymerisation process}

Semi-continuous emulsion copolymerisation of VAc-VEOVA 10 polymer were carried out using 0.5 -L five nicked round bottom flask equipped with a reflux condenser, stainless-steel stirrer and two separate feed streams. The first stream was a solution of pre-emulsion while the other feed stream was the initiator solution. The monomer mixture should be kept under continuous agitation throughout the polymerisation reaction. The mixture is placed under nitrogen, water and the anionic emulsifiers in the glass reactor and heated until $85^{\circ} \mathrm{C}$. Then, the water containing the initiator persulphate was added in the reactor. The reactor was heated to $85^{\circ} \mathrm{C}$ then $2.5 \%$ of pre-emulsion was added. The goal of this operation was to produce a seed. In this operation, only anionic emulsifier was used because non-ionic emulsifiers are less effective for particle nucleation. On the other hand, a small amount of butyl acrylate (BuAc) was used because it has been observed that polymerisation of VEOVA 10 starts much more readily in the presence of a small amount of BuAc (Unzué and Asua, 1993). After $10 \mathrm{~min}$, the rest of the pre-emulsion and initiator solution were then added in a period of $3 \mathrm{~h}$ (slowly at the beginning). After pre-emulsion addition and initiator solution are finished, the reactor was kept at $85^{\circ} \mathrm{C}$ for $2 \mathrm{~h}$ and aqueous solution of sodium meta-bisulphite and azobis were separately fed to the reactor during a 30 -min period to reduce the residual monomer. Then, the latex was cooled down to room temperature and was filtrated. 


\section{The factorial design}

In this paper, the authors are interested to study the effect of changing the feed composition of the VAc and VEOVA 10 (each at three levels) on four responses namely, MFFT; average [article diameter $(D)$; filtration residue and the cost. $3^{2}$ factorial design with four responses are used. In what follows, both the response factors as well as the controllable factors will be enlightened.

\subsection{The response factors}

\subsubsection{Average particle diameter (D)}

TEM of Zesis-type model EM.10, Germany, was used to measure the average particle diameter $(D)$ for the different polymer latex particles. The microscope was operated at $60 \mathrm{kV}$ with magnification ranges from 2500 to $5 \times 10^{5}$, and resolution of $0.5 \mathrm{~nm}$. Having a small average particle diameter which gave high binding power and high gloss of the emulsion film.

\subsubsection{Minimum film forming temperature (MFFT)}

MFFT is the temperature at which emulsion vehicles coalesce to form continuous films. Low temperature impairs the fluidity of the emulsion particle and thereby their ability to coalesce. Thus, emulsion that can withstand low temperatures is therefore preferable. MFFT is an important quality feature of emulsion vehicle that is determined by using a Rhopoint (manufactured in East Sussex, UK). MFFT 60 which is designed to conform to ASTM D2354 (1998).

\subsubsection{Filtration residue (ppm)}

This test method covers the determination of the filter-retained solids (grit) content of polymeric latex, i.e., material present in a latex specimen that is retained on a 200 -mech screen (ASTM D5097-90, 1996). Decreasing the filer-retained solids (ppm) results in a better performance polymer.

\subsubsection{Cost}

In addition to the above-mentioned three responses, the authors of this paper believe that the cost is a very vital factor that needs to be studied as a response factor. Without including the study of the cost, the experiment will be considered a lab experiment and not an applied one that can be taken to production. Doing so, the viability of the production of the polymer will be considering both the technical and the economical factors.

Having explained all the responses taken in this experiment, the next section explains the variation of the feed composition ratio for the controllable factors in the production of the polymer. 


\subsection{The controllable factors}

The feed composition of the controllable factors (VAc and VEOVA 10) is varied at three levels as shown in Table 1 , and its effect on the four responses is studied. The controllable factors are varied at only three levels to reduce the total number of experiments due to the fact that experiments are costly and time consuming. These ratios are the most common in literature within the study of the polymerisation process (Koukiotis and Sideridou, 2008). The amount of VAc and VEOVA 10 shown in Table 1 is measured in millilitres from the $200 \mathrm{ml}$ solution used in the experiment. The rest of the solution contains water, surfactant and initiator.

Table 1 Levels for the controllable factors

\begin{tabular}{lcccc}
\hline Factors & Low & Medium & High & Label \\
\hline VAc & 80 & 90 & 100 & VAc \\
VEOVA 10 & 20 & 12.5 & 5 & VEOVA 10 \\
\hline
\end{tabular}

\section{Statistical analysis and response surface methodology}

At each combination of the VAc and VEOVA 10 shown in Table 1, the four responses (MFFT, average particle diameter $(D)$, filtration residue $(\mathrm{ppm})$ and the cost) are measured (in case of the first three responses) or calculated (in case of the cost response) and are shown in Table 2. The cost of the polymer is calculated based on the average market price of the VAc and the VEOVA 10 (each litre of VAc is usually half the price of the VEOVA 10).

Table 2 The experimental data obtained (measured/calculated) for the four responses

\begin{tabular}{lccccccc}
\hline & \multicolumn{2}{c}{ Controllable factors } & & \multicolumn{4}{c}{ Response Factors } \\
\cline { 2 - 3 } \cline { 5 - 7 } Run & VAc $(\mathrm{ml})$ & VEOVA $10(\mathrm{ml})$ & & MFFT $\left({ }^{\circ} \mathrm{C}\right)$ & $D(\mathrm{~nm})$ & $\mathrm{ppm}$ & Cost $\times 10^{4}(\$)$ \\
\hline 1 & 80 & 20 & & 12.50 & 74.080 & 210.00 & 1200 \\
2 & 90 & 20 & & 12.80 & 80.200 & 240.00 & 1300 \\
3 & 100 & 20 & & 13.05 & 124.56 & 277.85 & 1400 \\
4 & 80 & 12.5 & & 13.30 & 145.45 & 345.00 & 1050 \\
5 & 90 & 12.5 & & 13.70 & 159.22 & 358.00 & 1150 \\
6 & 100 & 12.5 & & 13.90 & 167.65 & 369.87 & 1250 \\
7 & 80 & 5 & & 14.00 & 170.65 & 387.95 & 900 \\
8 & 90 & 5 & & 14.20 & 205.50 & 407.00 & 1000 \\
9 & 100 & 5 & & 14.80 & 263.74 & 438.00 & 1100 \\
\hline
\end{tabular}

The data in Table 2 was entered and analysed using Design Expert Software (2012). In the following subsections, the statistical analysis of the four responses will be performed to study the effect of changing the VAc and the VEOVA 10 on the four responses. Moreover, the values of the VAc and VEOVA 10 were the production should take place and should be determined to optimise the responses. 


\subsection{Minimum film forming temperature (MFFT)}

The first response that will be analysed is the MFFT. After entering the data into Design-Expert software, an ANOVA table (Table 3) is generated and residuals are plotted (Figures 1(a)-(c)).

Figure 1 (a), (b), (c) The residual plots for the MFFT (see online version for colours)

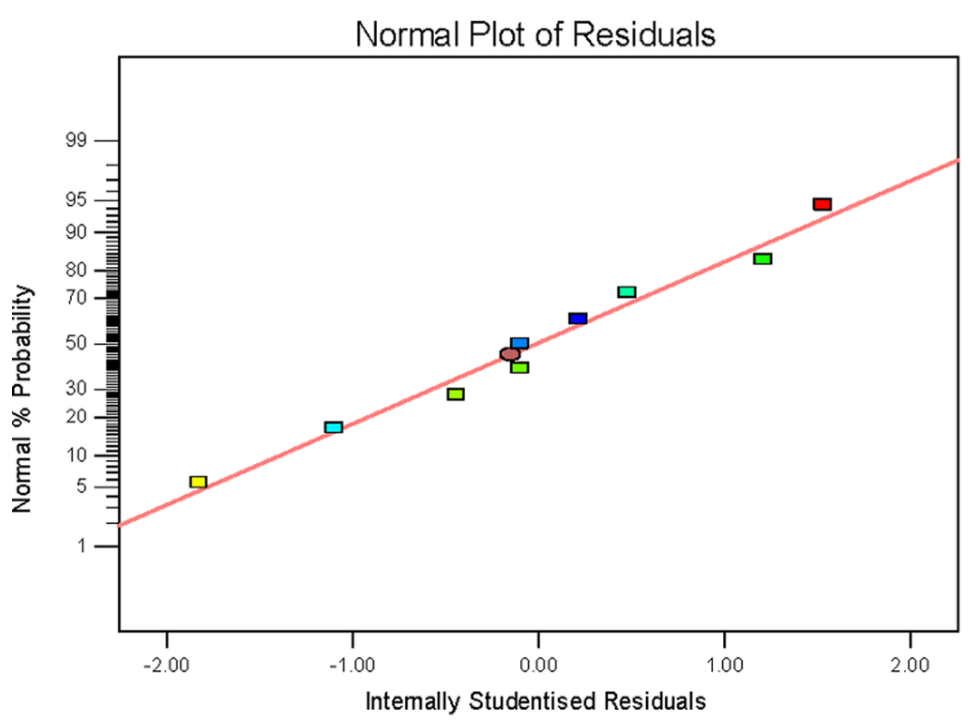

(a)

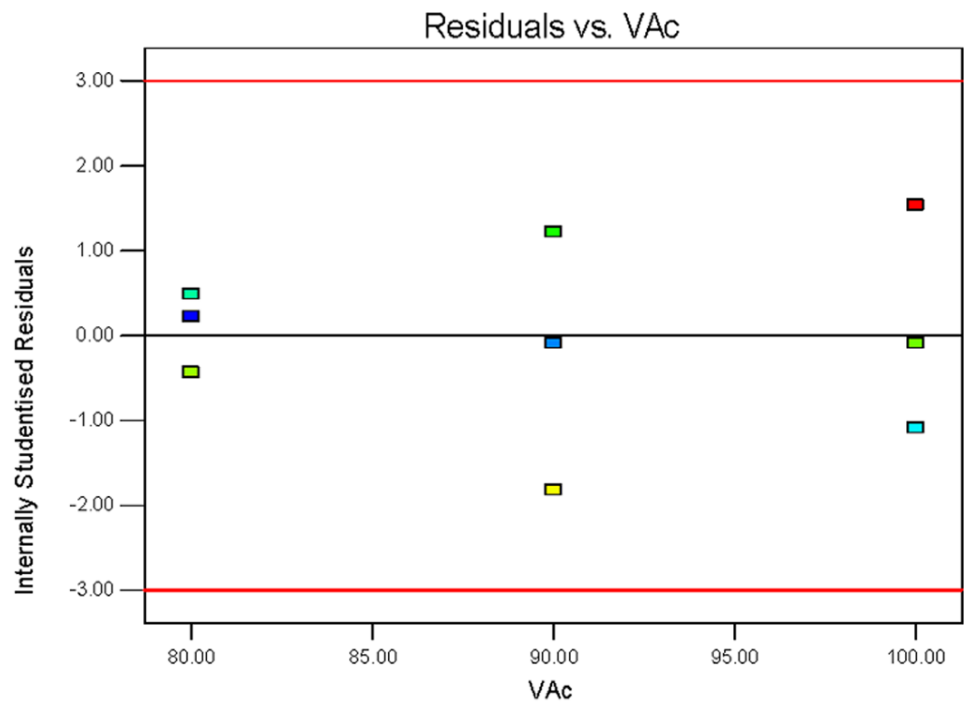

(b) 
Figure 1 (a), (b), (c) The residual plots for the MFFT (see online version for colours) (continued)

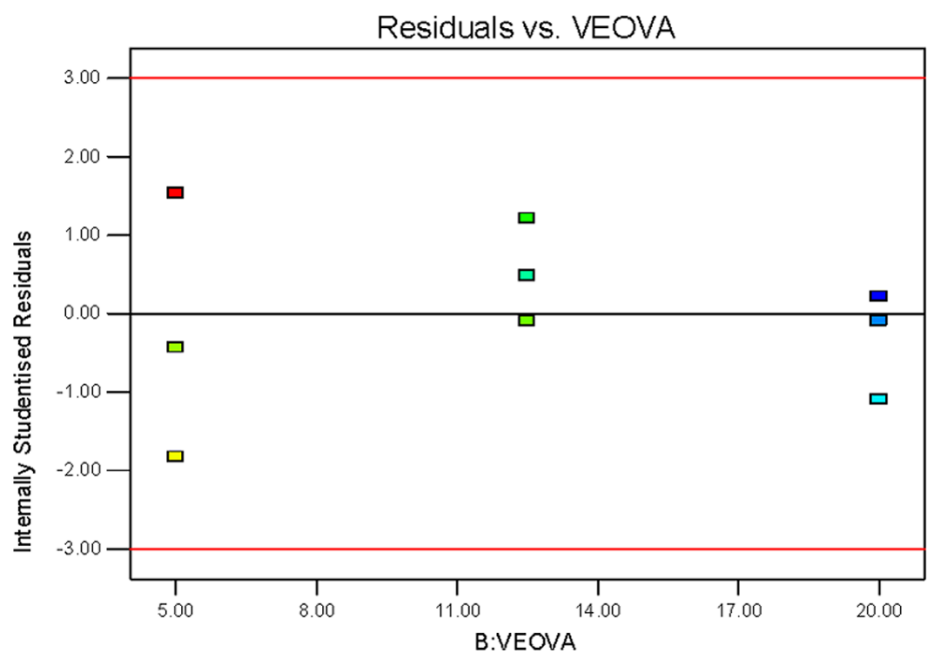

(c)

Table 3 Analysis of Variance for the MFFT

\begin{tabular}{lcccrr}
\hline Source & Sum of squares & Degrees offreedom & Mean square & F-value & $p$-value \\
\hline Model & 4.24 & 2 & 2.12 & 203.40 & $<0.0001$ \\
A-VAc & 0.63 & 1 & 0.63 & 60.84 & 0.0002 \\
B-VEOVA 10 & 3.60 & 1 & 3.60 & 345.96 & $<0.0001$ \\
Residual & 0.062 & 6 & 0.010 & & \\
Cor total & 4.30 & 8 & & & \\
\hline
\end{tabular}

If we assume that all analysis inferences are based on a significance level of $\alpha=0.05$, the $p$-value technique will be utilised to judge significance, i.e., if $p$-value $<\alpha$, then the factor is significant (Montgomery, 2010). The ANOVA table indicates clearly that both the VAc and the VEOVA 10 affects the MFFT. Moreover, the model $p$-value $(0.0001)<0.05$, indicates that the model is obviously significant. To investigate that the most significant factors in the process have been accounted for and that no nuisance or other factors were ignored, $R^{2}$ were calculated and found to be equal to 0.9855 . This $R^{2}$ value indicates adequate correlation between the response (MFFT) and the factors included in the design (VAc and VEOVA 10). Generally, values above 0.8 are acceptable. To trust the ANOVA table, the ANOVA assumptions need not to be violated. Thus, the residuals need to be visualised and analysed and ensured to follow normal distribution with constant variance. From that prospective, three residual plots were made, these are normal probability plot and the plot of residual against each factor of the controllable factors. These plots are shown in Figures 1(a)-(c).

As for the normal probability plot (Figure 1(a)), it can be noticed that all the residuals fall approximately on a straight line which confirm the assumptions that the residuals follow normal distribution. The other two plots confirm that the residuals have constant variance. This is concluded because the range between the residuals at each level of the controllable factor is approximately equal to the range in the other two levels. 
Same is concluded for the other controllable factor (Figures 1(b) and (c)). Thus it can be concluded that the ANOVA assumptions are not violated.

Figure 2 (a), (b) Contour plots and response surface for the MFFT (see online version for colours)

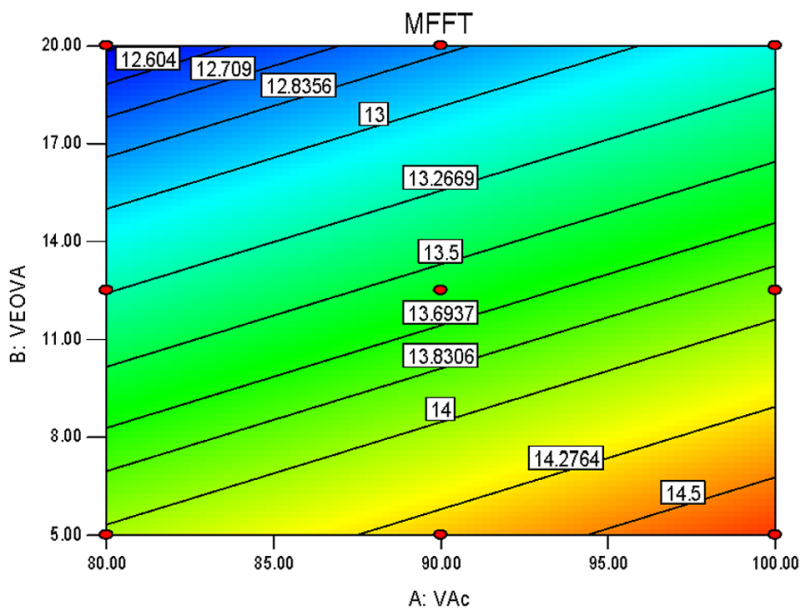

(a)

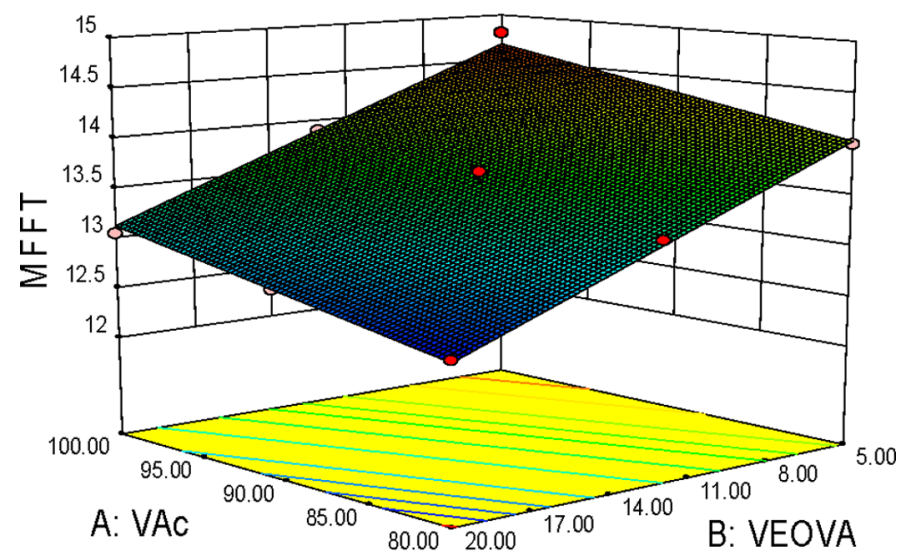

(b)

A secondary output from the Design-Expert software is the regression prediction model which is an expression of the response factor (MFFT) in terms of the significant effect factors (VAc and the VEOVA 10). The MFFT can be expressed as a function of the VAc and the VEOVA 10 as shown in equation (1)

$$
\mathrm{MFFT}=11.95+0.0325 \mathrm{VAc}-0.10333 \mathrm{VEOVA} 10
$$

This model can be used to determine the values of the VAc and the VEOVA 10 that will optimise (minimise) the MFFT. This is done through the establishment of the response surface and the contour plot of the MFFT which are shown in Figures 2(a) and (b). The response surface and the contour plots give insight on how to optimise the MFFT using the VAc and the VEOVA 10 factors. The MFFT decreases (improves) when the VAc is 
at the minimum level. In contrast a maximum level of VEOVA 10 decreases (improves) the MFFT response. Thus, to minimise the MFFT, the VAc and the VEOVA 10 should be kept at a level of 80 and 20, respectively. The contour plots and the response surface plot (Figures 2(a) and (b)) convey the same conclusion.

\subsection{Average particle diameter $(D)$}

The second response under study in this research is the average particle diameter $(D)$. The data in Table 2 corresponding to the average particle diameter $(D)$ has been entered into Design-Expert software. Using the software, ANOVA table (Table 4) is generated and residuals are plotted.

Table 4 Analysis of variance for the $D$

\begin{tabular}{lccccc}
\hline Source & Sum of squares & $D f$ & Mean square & F value & p-valueProb $>F$ \\
\hline Model & 26306.13 & 2 & 13153.07 & 47.82 & 0.0002 \\
A-VAc & 4579.95 & 1 & 4579.95 & 16.65 & 0.0065 \\
B-VEOVA 10 & 21726.18 & 1 & 21726.18 & 78.98 & 0.0001 \\
Residual & 1650.41 & 6 & 275.07 & & \\
Cor total & 27956.54 & 8 & & & \\
\hline
\end{tabular}

The ANOVA table shows that both the VAc and the VEOVA 10 affect the response factor (average particle diameter). This can be spotted from the fact that the $p$-value for both factors is less than 0.05 . The model is also significant ( $p$-value less than 0.05 ). To trust the ANOVA table, residual plots have been generated and revealed no violation for the ANOVA assumptions. The $R^{2}$ was calculated and found to be equal 0.9410, which is considered satisfactory and revealed that high power of correlation, it is presented in the model and that the most significant factors are involved in the model and no other significant factor is left behind.

The software also gives the regression prediction model that expresses the response factor $(D)$ as a function of the VAc and the VEOVA 10 as follows:

$$
D=6.20+2.76 \mathrm{VAc}-8.02 \text { VEOVA } 10
$$

This equation is utilised to construct the response surface and the contour plot as shown in Figures 3(a) and (b). These plot at then used to identify the values of the VAc and VEOVA 10 that maximise the response factor value $(D)$.

The contour plots and the response surface revealed the conclusion that in order to minimise the average particle diameter, VEOVA 10 should be kept at a value of 20 and VAc should be kept at a value of 80 . At that time the average particle diameter will be equal to 74.08 .

\subsection{Filtration residue (ppm)}

The last response to be evaluated in this research is the filtration residue (ppm). The ANOVA table for this response is shown in Table 5. 
Figure 3 (a), (b) Contour plots and response surface for the $D$ (see online version for colours)

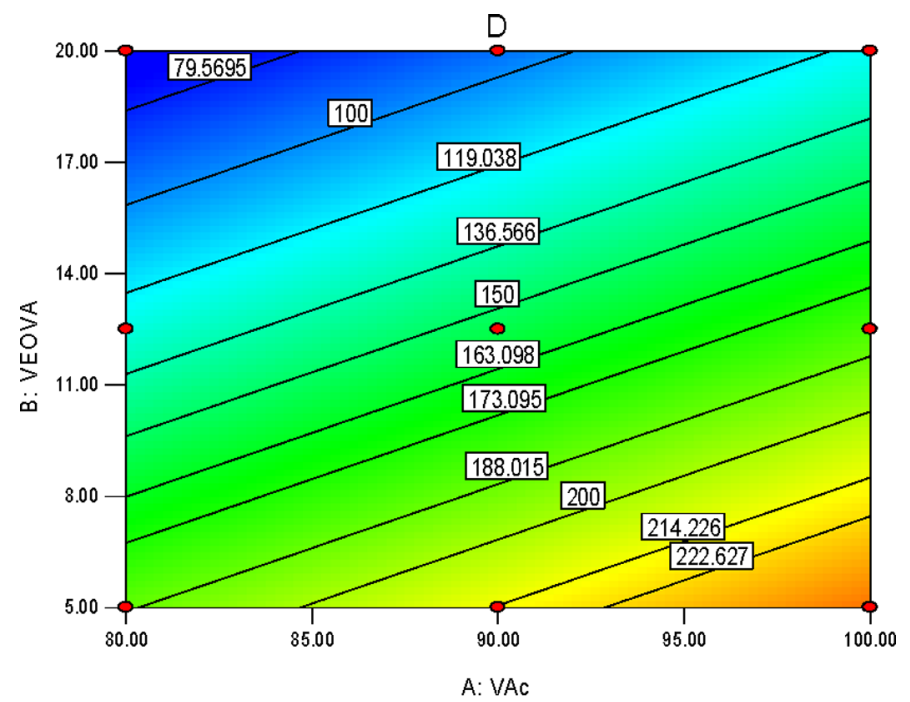

(a)

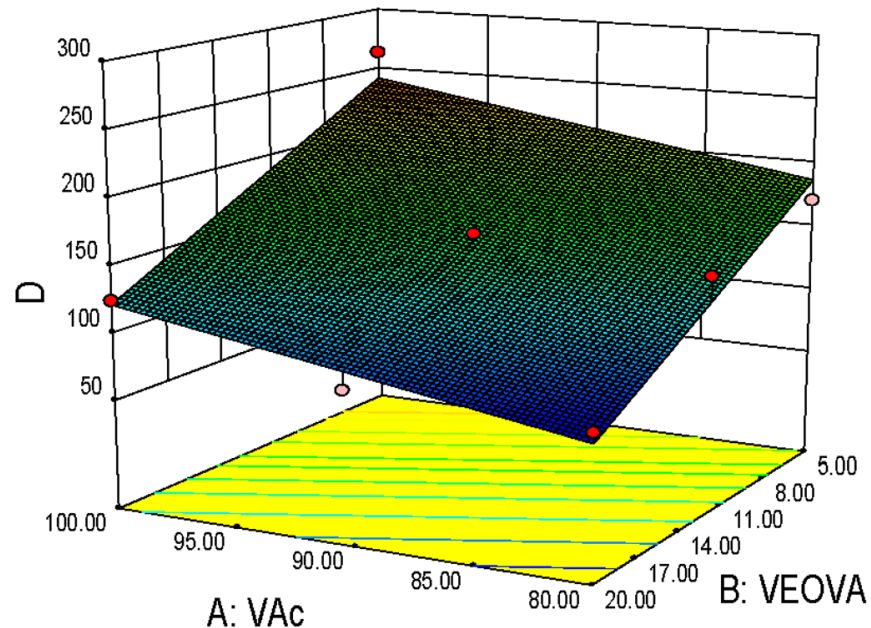

(b)

Table 5 Analysis of variance for the ppm

\begin{tabular}{lccccc}
\hline Source & Sum of squares & $D f$ & Mean square & $F$ value & p-valueProb $>F$ \\
\hline Model & 45918.21 & 2 & 22959.11 & 57.38 & 0.0001 \\
A-VAc & 3397.21 & 1 & 3397.21 & 8.49 & 0.0268 \\
B-VEOVA 10 & 42521.00 & 1 & 42521.00 & 106.27 & $<0.0001$ \\
Residual & 2400.80 & 6 & 400.13 & & \\
Cor total & 48319.01 & 8 & & & \\
\hline
\end{tabular}

Again the ANOVA table (Table 5) shows that both VAc and VEOVA 10 have significant effect on the ppm. Residual plots revealed no violation to the ANOVA assumptions. 
The plots show that the residual is normally and independently distributed with equal variance. The next step will be to generate the regression prediction model and $R^{2}$. The $R^{2}$ which was calculated and found to be equal to 0.9503 is considered satisfactory by the authors of this paper and gives indication of high power of correlation. In addition, the regression prediction model (equation (3)) is utilised to draw the response surface and the contour plots. Both the response surface and the contour plots revealed that the optimum (minimum) ppm can be achieved by keeping the VEOVA 10 at a value of 20 and the VAc at a level of 80

$$
\mathrm{ppm}=263.23+2.38 \mathrm{VAc}-11.22 \text { VEOVA } 10 .
$$

\subsection{Cost}

Although the above three responses achieved their optimum values by having the VEOVA 10 level kept at a value of 20 and the VAc level kept at a value of 80, taking this research to industrial application should involve the cost of producing the polymer. Thus, it is very important to study the effect of including the cost as an important response that will affect our decision. If we assumed that the market price of each $1000 \mathrm{~L}$ of VEOVA 10 costs $\$ 2000$ and each 1000 L of VAc costs $\$ 1000$, thus each $200 \mathrm{ml}$ of the polymer can be calculated as shown in equation (4):

$$
\text { Cost }\left(\times 10^{4}\right)=0.002(\operatorname{VEOVA} 10, \mathrm{ml})+0.001(\mathrm{VAc}, \mathrm{ml}) .
$$

Utilising equation (4), the cost of each $2000 \mathrm{~L}$ of the polymer (80 VAc-20 VEOVA) will cost $\$ 1200$. This includes the cost of the monomers only, used in the emulsion polymerisation process. It is worth saying that reducing VEOVA content only reduces the cost of the material. In addition, reducing VEOVA content will also reduce the cost of polymerisation due to the fact that less surfactant will be required to reach polymerisation. However, the reduction in the cost of the required surfactant is negligible as compared to the reduction in the VEOVA cost. For that reason, the cost of the polymer is calculated taking into account only the cost of the material of both the VAC and the VEOVA monomer, i.e., the main component in any emulsion recipe is the monomer material cost.

\section{Production optimisation}

In Section 4, each of the three responses and the cost are statistically analysed separately. However, most of the times, the decision maker will require the optimisation of all the responses simultaneously. This will be done by optimising equations (1)-(4). In such case, the decision maker must specify the relative importance of each response from his prospective. It is quite clear that changing the relative importance might change the values of the controllable factors (VAc and VEOVA 10) that optimise the responses. In this research, two cases of relative importance are considered that are shown in Table 6 together with the optimum values of the controllable factors that optimise the response factors according to their relative importance. The optimum response values are also shown in the same table. The relative importances are shown as a score from one to five with one as the lowest importance and five as the highest importance. All the optimisation calculations are performed using the optimisation module of the 
Design Expert Software (2012). In Table 6, the authors only changed the relative importance of the cost (maximum and minimum) to show its effect on the overall design. It has to be noted that our goal is not just to reduce cost otherwise we could have taken that design which costs only $\$ 900$. However, this design highly jeopardise the quality of the output emulsion. In fact, our goal is to minimise cost while also taking other design parameters into consideration to avoid deterioration of the emulsion quality used for different application.

Table 6 Decision making optimisation matrix

\begin{tabular}{lccccc}
\hline Case & Response factors & Relative importance & Response values & VAc & VEOVA 10 \\
\hline Case 1 & MFFT & 1 & 12.62 & 80 & 17.31 \\
& $D$ & 5 & 78.17 & & \\
& ppm & 3 & 244.99 & & \\
& Cost & 5 & 1171.67 & & \\
\hline Case 2 & MFFT & 1 & 12.48 & 80 & 20 \\
& $D$ & 5 & 66.76 & & \\
& ppm & 3 & 229.10 & & \\
& Cost & 1 & 1200 & & \\
\hline
\end{tabular}

It can be concluded from Table 6 that giving the cost high relative importance, reduces the cost of producing $2000 \mathrm{~L}$ of the polymer by $2.4 \%$ which is considered a significant reduction in cost especially in the very competitive market of the polymer production.

According to expert opinion in the field of emulsions, the above reduction in cost is much more worthier than deterioration (if any) in the emulsion quality and its application.

\section{Conclusion}

This paper studies the influential factors that affect the production of the emulsion. These include the amount of VAc and VEOVA 10 monomers on the average particle diameter $(D)$, MFFT, the filtration residue (ppm) and the cost. It can be concluded from the paper that the average particle diameter of the emulsion $(D)$ decreases with the increase of the amount of VEOVA 10 content (and subsequently decrease the amount of VAc), also the ppm and MFFT decrease in this case. Including the cost as a response reduces the cost of the production of the polymer by $2.4 \%$ which is considered a significant reduction due to the competitive nature of the polymer production market.

\section{References}

Allen, G. and Bevington, J.C. (1989) Comprehensive Polymer Science: The Synthesis, Characterization, Reactions and Applications of Polymers, Vol. 4, Chain Polymerization Part 2.

ASTM D2354-1998 (1998) Standard Test Method for Minimum Film Formation Temperature (MFFT) of Emulsion Vehicles.

ASTM D5097-90 (1996) Standard Test Method for Filter-Retained Solids Content of Polymer Latexes (Reapproved 1996). 
Design Expert Software (2012), Statease Incorporation, Version 8.0.7.1.

Fitch, R.M. (1997) Polymer colloids: A comprehensive Introduction, Academic Press, New York.

Kondo, A., Kamura, H. and Higashitani, K. (1994) 'Development and application of thermo-sensitive magnetic immunomicrospheres for antibody purification', Appl. Microbiol. Biotechnol., Vol. 41, No. 1, pp.99-105.

Koukiotis, C. and Sideridou, I.D. (2008) 'Preparation of high solids stable translucent nanolatexes of MMA/BA copolymers and MMA/BA/Veova-10 terpolymers with low MFFT using green industrial surfactants', Progress in Organic Coatings, Vol. 63, pp.116-122.

Lovell, P. and El-Asser, M. (1997) Emulsion Polymerization and Emulsion Polymers, John Wiley\& Sons Inc, New York.

Montgomery, D.C. (2010) Design and Analysis of Experiments, John Wiley \& Sons Inc., Singapore.

Niu, M., Du, M., Gao, Z., Yang, C., Lu, X., Qiao, R. and Gao, M. (2010) 'Monodispersed magnetic polystyrene beads with excellent colloidal stability and strong magnetic response', Macromol. Rapid Commun., Vol. 31, pp.1805-1810.

Unzué, M.J. and Asua, J.M. (1993) 'Semicontinuous miniemulsion terpolymerization: effect of the operation conditions', J. Appl. Polym. Sci., Vol. 49, pp.81-90.

Urban, D. and Takamura, K. (2002) Polymer Dispersions and their Industrial Applications, Wiley-VCH, Weinheim.

Zhao, W., Shi, J., Chen, H. and Zhang, L. (2006) 'Particle size, uniformity, and mesostructure control of magnetic core/mesoporous silica shell nano-composite spheres', Journal of Materials Research, Vol. 21, No. 12, pp.3080-3089.

\section{Website}

URL: http://www.klinegroup.com/brochures/v359/brochure.pdf 